\title{
Protect your lungs: get vaccinated
}

\author{
Getting vaccinated can protect you from lots of \\ different diseases and help you keep your lungs healthy
}
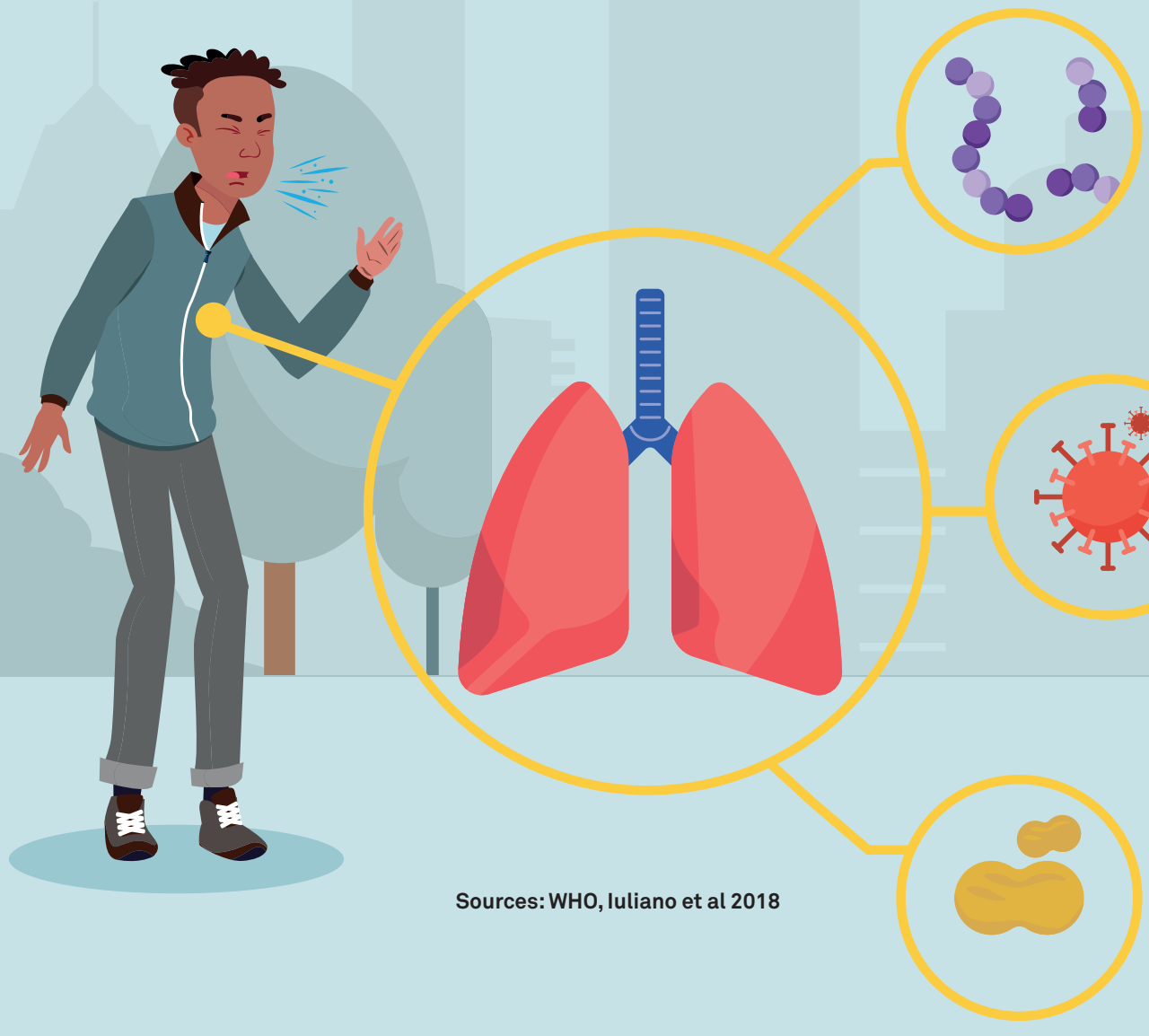

Sources: WHO, Iuliano et al 2018

\section{Pneumococcus}

Pneumococcus can lead to

pneumonia - a severe lung infection.

1.6 million deaths each year

\section{Whooping cough}

A serious cough that can last for months and mainly affects children. Damage caused by the virus can also lead to bronchiectasis - a severe and long-term lung disease.

\section{0,000 deaths each year}

\section{Vaccination can also help to protect other people}

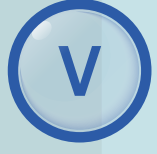

Vaccinated

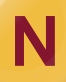

Not able to be

vaccinated

People can be protected if those close

to them (like friends and family members) and enough people in their communities (including healthcare professionals) are vaccinated, because it stops diseases from spreading. 


\section{Vaccination is important if you have a lung disease or other health condition}

People with a lung condition or other health conditions can be at a higher risk from lung infections. You can prevent some of these infections by getting vaccinated. Consider which vaccines you should have and when you should have them, together with your healthcare professional.

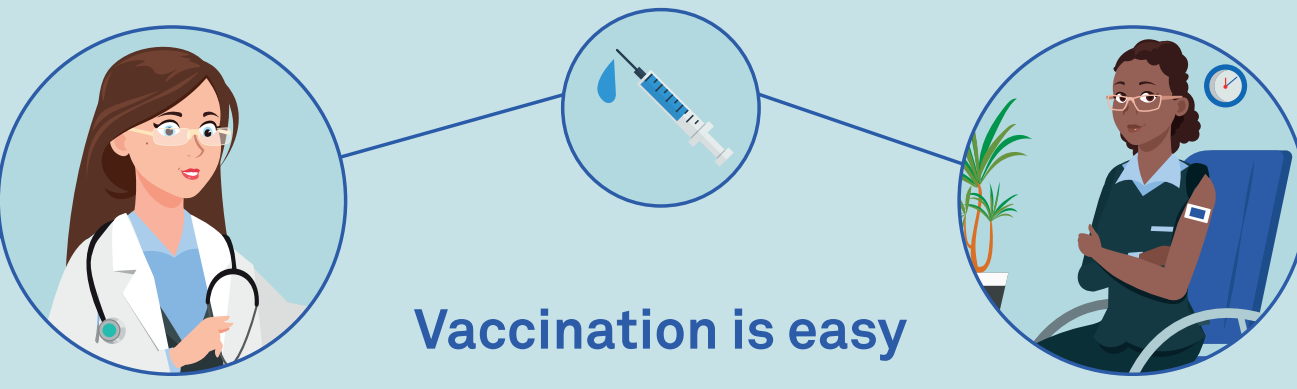

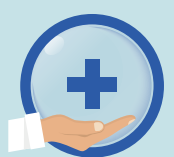

The healthcare professional will clean the skin...

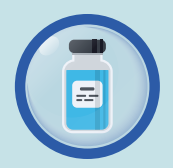

and inject a small amount of liquid into the muscle, usually at the top of the arm.

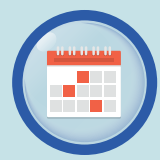

To protect you against some diseases, you may need to go back for a second or third vaccination.

\section{Major* side effects from vaccinations are very rare}

\section{If everyone in each of these cities were vaccinated, less than \\ one person per city would experience a major side effect.}

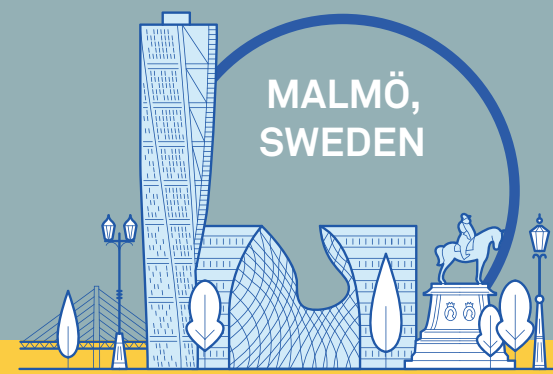

339,313 people

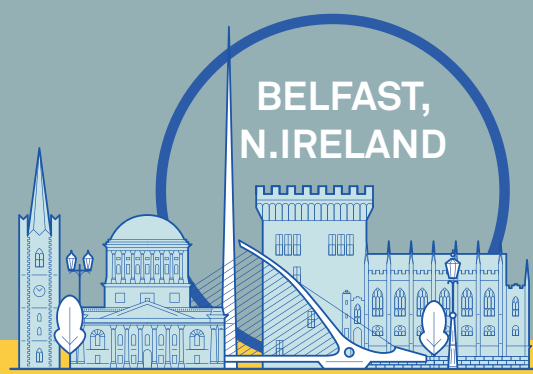

342,637 people

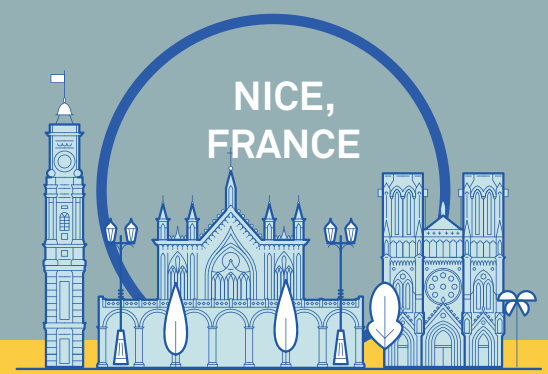

340,200 people
Sources

1. WHO 2019 Pneumococcal disease https://www.who.int/ 2. WHO 2018 Influenza (Seasonal) https://www.who.int/ 3. Iuliano et al. 2018. Estimates of global seasonal influenza-associated respiratory mortality: a modelling study. The Lancet. 4. WHO 2018 Pertussis https://www.who.int/ 5. Liang et al. 2011. Safety of Influenza A (H1N1) vaccine in postmarketing surveillance in China; N Engl J Med. 6. Esposito et al 2018 Incidence of outcomes relevant to vaccine safety monitoring in a US commercially-insured population. Vaccine. 7. Xu et al 2019 The effectiveness and safety of pertussis booster vaccination for adolescents and adults. Medicine. 8. ONS 2017 Population Estimates for UK, England and Wales, Scotland and Northern Ireland https://www.ons.gov.uk/ 9. INSEE 2015 Populations légales 2015 https://insee.fr/ 10. Statistics Sweden 2019 Population 2018-12-31 by region, municipal. https://www.scb.se/

\section{(x) HEALIHY LUNGS FOR LIFE} OERS ELF

Find out more at:

www.healthylungsforlife.org
This document was produced with the aim of helping healthcare professionals explain the benefits of being vaccinated to their patients. It was produced by the European Lung Foundation (ELF) as part of the Healthy Lungs for Life campaign.

* Major side effects are those that are long lasting or permanent, for example immune disorders.

Produced in July 2019

Republished in September 2019 to correct an error in the mortality statistics (page 1) 